Pacific Journal of Mathematics

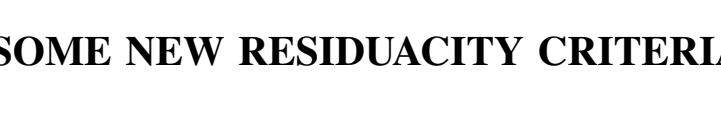




\section{SOME NEW RESIDUACITY CRITERIA}

\section{Richard H. Hudson and KenNeth S. Williams}

Let $e$ and $k$ be integers $\geq 2$ with $e$ odd and $k$ even. Set $2 l=\mathrm{L}$. C. M. $(e, k)$ and let $p$ be a prime with $p \equiv 1(\bmod 2 l)$ having $g$ as a primitive root. It is shown that the index of $e$ (with respect to $g$ ) modulo $k$ can be computed in terms of the cyclotomic numbers of order $l$. By applying this result with $e=3, k=4 ; e=5, k=4 ; e=3, k=8$; new criteria are obtained for 3 and 5 to be fourth powers $(\bmod p)$ and for 3 to be an eighth power $(\bmod p)$.

1. Introduction. Let $e$ and $k$ be integers greater than or equal to 2 with $e$ odd and $k$ even. Let $p$ be a prime congruent to 1 modulo $2 l$, where $2 l=$ L.C.M. $(e, k)$. Let $g$ be a fixed primitive root $(\bmod p)$. If $a$ is an integer not divisible by $p$, the index of $a$ with respect to $g$ is denoted by ind $(a)$ and is the least nonnegative integer $b$ such that $a \equiv g^{b}(\bmod p)$. For $0 \leqq h, k \leqq l-1$, the cyclotomic number $(h, k)_{l}$ of order $l$ is the number of integers $n$ $(1 \leqq n \leqq p-2)$ such that ind $(n) \equiv h(\bmod l)$, ind $(n+1) \equiv k(\bmod l)$.

Using an idea due to Muskat [4: 257-258], we prove the following congruence for the index of $e$ modulo $k$.

THEOREM 1.

$$
\begin{aligned}
\text { ind }(e) \equiv & 2 \sum_{i=1}^{k / 2-1} i \sum_{j=1}^{(e-1) ! 2} \sum_{r=0}^{2 l / k-1} \sum_{s=0}^{l / e-1}\left(i+r \cdot \frac{k}{2}, j+s e\right)_{l} \\
& +\frac{(p-1)(e-1)^{2}}{8 e}(\bmod k) .
\end{aligned}
$$

Applying Theorem 1 with $e=3, k=4$, we obtain the following criterion for 3 to be a fourth power $(\bmod p)$.

THEOREM 2. Let $p \equiv 1(\bmod 12)$ be a prime, so that there are integers $x$ and $y$ satisfying

$$
p=x^{2}+3 y^{2}, \quad x \equiv 1(\bmod 3) .
$$

Then 3 is a fourth power $(\bmod p)$ if and only if $x \equiv 1(\bmod 4)$.

This criterion should be compared with the classical result: 3 is a fourth power $(\bmod p)$ if and only if

$$
\begin{cases}b \equiv 0(\bmod 3), & \text { if } \quad p \equiv 1(\bmod 24) \\ a \equiv 0(\bmod 3), & \text { if } \quad p \equiv 13(\bmod 24)\end{cases}
$$


where

$$
p=a^{2}+b^{2}, \quad a \equiv 1(\bmod 4), \quad b \equiv 0(\bmod 2),
$$

see for example [2: p. 24].

Next taking $e=5, k=4$, in Theorem 1 we obtain the following new criterion for 5 to be a fourth power $(\bmod p)$.

THEOREM 3. Let $p \equiv 1(\bmod 20)$ be a prime, so that there are integers $x, u, v$, and $w$ satisfying

$$
16 p=x^{2}+50 u^{2}+50 v^{2}+125 w^{2}, \quad x w=v^{2}-4 u v-u^{2},
$$

and

$$
x \equiv 1(\bmod 5) .
$$

Then 5 is a fourth power $(\bmod p)$ if and only if

$$
\begin{cases}x \equiv 4(\bmod 8), & \text { if } x \equiv 0(\bmod 2), \\ x \equiv \pm 3 w(\bmod 8), & \text { if } x \equiv 1(\bmod 2) .\end{cases}
$$

This criterion should be compared with the well-known result (see for example [2: p. 24]):

5 is a fourth power $(\bmod p)$ if and only if $b \equiv 0(\bmod 5), \quad$ where $p=a^{2}+b^{2}, \quad a \equiv 1(\bmod 4), \quad b \equiv 0(\bmod 2)$.

Finally, applying Theorem 1 with $e=3, k=8$, we obtain the following new criterion for 3 to be an eighth power $(\bmod p)$.

THEOREM 4 . Let $p \equiv 1(\bmod 24)$ be a prime so that there are integers $a, b, x$ and $y$ satisfying

$$
p=a^{2}+b^{2}=x^{2}+3 y^{2},
$$

and

$$
a \equiv 1(\bmod 4), \quad b \equiv 0(\bmod 4), \quad x \equiv 1(\bmod 6), \quad y \equiv 0(\bmod 2) .
$$

Assume 3 is a fourth power $(\bmod p)$, so that

$$
b \equiv 0(\bmod 3), \quad x \equiv 1(\bmod 4) .
$$

Then 3 is an eighth power $(\bmod p)$ if and only if

$$
a \equiv 1(\bmod 3), \quad y \equiv 0(\bmod 8),
$$

or

$$
a \equiv-1(\bmod 3), \quad y \equiv 4(\bmod 8)
$$


This criterion should be compared to that of von Lienen [3: $p$. 114], namely, if 3 is a fourth power $(\bmod p)$ then 3 is an eighth power $(\bmod p)$ if and only if

$$
\begin{cases}a \equiv c(\bmod 3), & \text { if } \quad p \equiv 1(\bmod 48), \\ a \equiv-c(\bmod 3), & \text { if } \quad p \equiv 25(\bmod 48),\end{cases}
$$

where

$$
p=a^{2}+b^{2}=c^{2}+2 d^{2}
$$

and

$a \equiv 1(\bmod 4), \quad b \equiv 0(\bmod 4), \quad c \equiv 1(\bmod 4), \quad d \equiv 0(\bmod 2)$.

Combining these results, we see that if $(3 / p)_{4}=+1$ (equivalently $b \equiv 0(\bmod 3)$ or $x \equiv 1(\bmod 4))$, we have

$$
\left\{\begin{array}{lll}
y \equiv 0(\bmod 8) \Longleftrightarrow c \equiv 1(\bmod 3), & \text { if } & p \equiv 1(\bmod 48), \\
y \equiv 0(\bmod 8) \Longleftrightarrow c \equiv-1(\bmod 3), & \text { if } & p \equiv 25(\bmod 48) .
\end{array}\right.
$$

2. Proof of Theorem 1. The roots of the congruence

$$
\frac{x^{e}-1}{x-1} \equiv 0(\bmod p)
$$

are

$$
x \equiv g^{j f}(\bmod p), \quad j=1,2, \cdots, e-1,
$$

where $p-1=e f$, so that

$$
x^{e-1}+x^{e-2}+\cdots+x+1 \equiv \prod_{j=1}^{e-1}\left(x-g^{j f}\right)(\bmod p) .
$$

Taking $x=1$ in (2.2), we obtain

$$
e \equiv \prod_{j=1}^{e-1}\left(1-g^{j f}\right)(\bmod p),
$$

and so

$$
\text { ind }(e) \equiv \sum_{j=1}^{e-1} \text { ind }\left(1-g^{j f}\right)(\bmod p-1) \text {. }
$$

Next

$$
\begin{aligned}
& \sum_{j=(e+1) / 2}^{e-1} \text { ind }\left(1-g^{j f^{j}}\right) \\
&=\sum_{j=1}^{(e-1) / 2} \text { ind }\left(1-g^{(e-j) f}\right)
\end{aligned}
$$




$$
\begin{aligned}
& =\sum_{j=1}^{(e-1) / 2} \text { ind }\left(1-g^{-j f}\right) \\
& \equiv \sum_{j=1}^{(e-1) / 2} \text { ind }\left(1-g^{j f}\right)+\sum_{j=1}^{(e-1) / 2} \text { ind }\left(-g^{-j f}\right)(\bmod p-1) \\
& \equiv \sum_{j=1}^{(e-1) / 2} \text { ind }\left(1-g^{j f}\right)+\sum_{j=1}^{(e-1) / 2}\left(\frac{p-1}{2}-j f\right)(\bmod p-1),
\end{aligned}
$$

so

(2.5) $\quad$ ind $(e) \equiv 2 \sum_{j=1}^{(e-1) ! 2}$ ind $\left(1-g^{j f}\right)+\frac{(p-1)(e-1)^{2}}{8 e}(\bmod p-1)$.

Next the roots of

$$
x^{f}-g^{j f} \equiv 0(\bmod p)
$$

are

$$
x \equiv g^{e i+j}(\bmod p) \quad(i=1,2, \cdots, f),
$$

so

$$
x^{f}-g^{j f} \equiv \prod_{i=1}^{f}\left(x-g^{e i+j}\right)(\bmod p) .
$$

Taking $x=1$ in (2.6), we obtain

$$
1-g^{j f} \equiv \prod_{i=1}^{f}\left(1-g^{e i+j}\right)(\bmod p)
$$

so

$$
\text { ind }\left(1-g^{j f}\right) \equiv \sum_{i=1}^{f} \operatorname{ind}\left(1-g^{e i+j}\right)(\bmod p-1) \text {. }
$$

Further, working modulo $k / 2$, we have

$$
\begin{aligned}
& \sum_{i=1}^{f} \text { ind }\left(1-g^{e i+j}\right) \\
& =\sum_{\substack{n=2 \\
\operatorname{ind}(n) \triangleq j(\bmod e)}}^{p-1} \text { ind }(1-n) \\
& \equiv \sum_{\substack{n=2 \\
\operatorname{ind}(n) \equiv j(\bmod e)}}^{p-1} \text { ind }(n-1)+\sum_{\substack{n=2 \\
\operatorname{ind}(n) \equiv j(\bmod e)}}^{p-1} \text { ind }(-1) \\
& \equiv \sum_{\substack{n=1 \\
\operatorname{ind}(n+1) \equiv j(\bmod e)}}^{p-2} \text { ind }(n)+\frac{p-1}{2} \sum_{\substack{n=1 \\
\text { ind } n \equiv j(\bmod e)}}^{p-1} 1 \\
& \equiv \sum_{\substack{i=0 \\
\text { ind }(n) \equiv i(\bmod k / 2)}}^{k / 2-1} \sum_{\substack{n=1 \\
\operatorname{ind}(n+1) \equiv j(\bmod e)}}^{p-2} i,
\end{aligned}
$$

that is 


$$
\sum_{i=1}^{f} \operatorname{ind}\left(1-g^{e++j}\right) \equiv \sum_{i=1}^{k / 2-1} i \sum_{r=0}^{2 l^{\prime} k-1} \sum_{s=0}^{l / e-1}(i+r k / 2, j+s e)_{l} .
$$

The result now follows from (2.5), (2.7) and (2.8).

3. Proof of Theorem 2. Taking $e=3, k=4$, so that $l=6$, in Theorem 1 , we obtain, for $p \equiv 1(\bmod 12)$,

$$
\text { ind }(3) \equiv 2 \sum_{r=0}^{2} \sum_{s=0}^{1}(1+2 r, 1+3 s)_{6}+\frac{p-1}{6}(\bmod 4) .
$$

Defining $x$ and $y$, as in [6: p. 68], by

$$
x=6(0,3)_{6}-6(1,2)_{6}+1
$$

and

$$
y=(0,1)_{6}-(0,5)_{6}-(1,3)_{6}+(1,4)_{6},
$$

so that $x$ and $y$ satisfy (1.1), from the tables for the cyclotomic numbers of order 6 , we obtain

$$
\sum_{r=0}^{2} \sum_{s=0}^{1}(1+2 r, 1+3 s)_{6}=\frac{1}{6}(p-x-3 y) .
$$

Hence, from (3.1), we obtain

$$
\text { ind }(3) \equiv \frac{1}{3}(p-x)-y+\frac{p-1}{6}(\bmod 4) \text {. }
$$

Now

$$
y \equiv \begin{cases}0(\bmod 4), & \text { if } \quad p \equiv 1(\bmod 24) \\ 2(\bmod 4), & \text { if } \quad p \equiv 13(\bmod 24)\end{cases}
$$

that is

$$
y \equiv \frac{1}{6}(p-1)(\bmod 4),
$$

giving

$$
\text { ind }(3) \equiv \frac{1}{3}(p-x) \equiv \frac{1}{3}(1-x)(\bmod 4) \text {, }
$$

which completes the proof of Theorem 2 .

4. Proof of Theorem 3. Taking $e=5, k=4$, so that $l=10$, in Theorem 1 , we obtain for $p \equiv 1(\bmod 20)$, 


$$
\text { ind }(5) \equiv 2 \sum_{j=1}^{2} \sum_{r=0}^{4} \sum_{s=0}^{1}(1+2 r, j+5 s)_{10}+\frac{2}{5}(p-1)(\bmod 4) \text {. }
$$

Define $m$ by $2 \equiv g^{m}(\bmod p)$. Replacing $g$ by an appropriate power of $g$, we may suppose that $m \equiv 0$ or $1(\bmod 5)$. Next we define $x$, $u, v, w$ by

$$
\begin{aligned}
3 x & =-p+14+25(0,0)_{5}, \\
u & =(0,2)_{5}-(0,3)_{5}, \\
v & =(0,1)_{5}-(0,4)_{5}, \\
w & =(1,3)_{5}-(1,2)_{5},
\end{aligned}
$$

so that $x, u, v, w$ is a solution of (1.2) satisfying (1.3) (see for example [5: p. 100]). From the tables of Whiteman [5: pp. 107-109] for the cyclotomic numbers of order 10, we obtain in the case $m \equiv 0(\bmod 5)$, that is, 2 is a fifth power $(\bmod p)$ or equivalently, $x \equiv 0(\bmod 2)$ [1: p. 13]:

$$
\begin{aligned}
\sum_{j=1}^{2} \sum_{r=0}^{4} \sum_{s=0}^{1}(1 & +2 r, j+5 s)_{10} \\
& =\frac{1}{20}\{4 p+x-15 u+15 v-30 w\},
\end{aligned}
$$

so

$$
\text { ind } \begin{aligned}
(5) & \equiv \frac{1}{10}\{4 p+x-15 u+15 v-30 w\}(\bmod 4) \\
& \equiv \frac{1}{10}(x+4)-\frac{3}{2}(u-v)+w(\bmod 4)
\end{aligned}
$$

Emma Lehmer [1: p. 13] has shown in this case that

$$
x \equiv u \equiv v \equiv w \equiv 0(\bmod 4), \quad u \equiv v(\bmod 8),
$$

so that

$$
\text { ind }(5) \equiv \frac{1}{10}(x+4) \equiv \frac{x}{2}+2(\bmod 4),
$$

completing the proof of Theorem 3 in this case.

When $m \equiv 1(\bmod 5), 2$ is not a fifth power $(\bmod p)$ and $x \equiv 1(\bmod 2)$. From the tables of Whiteman [5: pp. 107-109], in this case, we obtain

$$
\begin{aligned}
& \sum_{j=1}^{2} \sum_{r=0}^{4} \sum_{s=0}^{1}(1+2 r, j+5 s)_{10} \\
& \quad=\frac{1}{40}\{8 p-3 x+10 u+20 v-25 w\},
\end{aligned}
$$


so that

$$
4 \text { ind }(5) \equiv 8 p-3 x+10 u+20 v-25(\bmod 16),
$$

which shows that $w \equiv 1(\bmod 2)$.

Since

$$
400(0,2)_{10}=4 p-36+17 x+50 u-25 w,
$$

we have $(\operatorname{as} x \equiv w \equiv 1(\bmod 2))$

$$
10 u \equiv 3 x+5 w(\bmod 16),
$$

so that

$$
\text { ind }(5) \equiv v+w(\bmod 4) \text {. }
$$

As

$$
200(0,9)_{10}=2 p-18-4 x+25 u-25 v+25 w
$$

and

$$
200(1,2)_{10}=2 p+2+x+25 u+25 v-50 w
$$

we have

$$
\left\{\begin{array}{l}
u-v \equiv 4-w(\bmod 8) \\
u+v \equiv 4+2 w-x(\bmod 8)
\end{array}\right.
$$

so

$$
u \equiv \frac{1}{2}(w-x)(\bmod 4), \quad v \equiv \frac{1}{2}(3 w-x)(\bmod 4) .
$$

Hence we have

$$
\text { ind }(5) \equiv \frac{1}{2}(5 w-x)(\bmod 4) \text {. }
$$

Since all solutions of (1.2) satisfying (1.3) are given by (see for example [1: p. 13])

$$
(x, u, v, w), \quad(x, v,-u,-w), \quad(x,-u,-v, w), \quad(x,-v, u,-w),
$$

(4.2) gives

$$
\text { ind }(5) \equiv 0(\bmod 4) \Longleftrightarrow x \equiv \pm 3 w(\bmod 8) \text {, }
$$

and

$$
\text { ind }(5) \equiv 2(\bmod 4) \Longleftrightarrow x \equiv \pm w(\bmod 8),
$$

which completes the proof of Theorem 3. 
5. Proof of Theorem 4. Taking $e=3, k=8$ so that $l=12$, in Theorem 1 , we obtain, for $p \equiv 1(\bmod 24)$,

$$
\text { ind }(3) \equiv 2 \sum_{i=1}^{3} i \sum_{r=0}^{2} \sum_{s=0}^{3}(i+4 r, 1+3 s)_{12}+\frac{1}{6}(p-1)(\bmod 8) \text {. }
$$

Following Whiteman [6: p. 64], we define $m$ and $m^{\prime}$ by $2 \equiv g^{m}(\bmod p)$ and $3 \equiv g^{m^{\prime}}(\bmod p)$ respectively. As $p \equiv 1(\bmod 8)$ we have $m \equiv 0(\bmod 2)$. Replacing $g$ by an appropriate power of $g$ we may suppose that $m \equiv 0$ or $2(\bmod 3)$, so that $m \equiv 0$ or $2(\bmod 6)$. Further, as we are assuming 3 is a fourth power $(\bmod p)$, we have $m^{\prime} \equiv 0(\bmod 4)$. Next we define $x$ and $y($ as in [6: p. 68]) by

$$
\begin{aligned}
& x=6(0,3)_{6}-6(1,2)_{6}+1, \\
& y=(0,1)_{6}-(0,5)_{6}-(1,3)_{6}+(1,4)_{6},
\end{aligned}
$$

and $a$ and $b$ by equations (4.4) and (4.5) in [6] (a replaces Whiteman's $x, b$ replaces Whiteman's 2y). Then $x, y, a, b$ satisfy (1.4) and (1.5). Whiteman [6: pp. 69-73] gives the cyclotomic numbers of order 12 in terms of $x, y, a$ and $b$, as defined above. When $m \equiv 0(\bmod 6)$, we must use Tables 9 and 10 of [6] and, when $m \equiv 2(\bmod 6)$, we must use Tables 3 and 4 . By considering the cyclotomic numbers $(3,6)_{12}$ in Table $9 ;(2.4)_{12}$ in Table $10 ;(1,2)_{12}$ in Table $3 ;(2,8)_{12}$ in Table 4; it is easy to check that Whiteman's quantity $c= \pm 1$ (see [6: pp. 64-65]) satisfies

$$
\left\{\begin{array}{l}
c=+1 \Longleftrightarrow a \equiv 1(\bmod 3), \\
c=-1 \Longleftrightarrow a \equiv 2(\bmod 3) .
\end{array}\right.
$$

We remark that $a \not \equiv 0(\bmod 3)$ as 3 is assumed to be a fourth power $(\bmod p)$.

Next we set

$$
\sum_{i}=\sum_{r=0}^{2} \sum_{s=0}^{3}(i+4 r, 1+3 s)_{12} \quad(i=1,2,3),
$$

so that

$$
\text { ind }(3) \equiv 2\left(\sum_{1}+2 \sum_{2}+3 \sum_{3}\right)+\frac{1}{6}(p-1)(\bmod 8) \text {. }
$$

From Whiteman's tables, we obtain

$$
\begin{aligned}
12 \sum_{1} & = \begin{cases}p-2 b-x-3 y, & \text { if } \quad a \equiv 1(\bmod 3), \\
p+2 b-x-3 y, & \text { if } \quad a \equiv-1(\bmod 3),\end{cases} \\
12 \sum_{2} & =\left\{\begin{array}{lll}
p-2 a+x+3 y, & \text { if } \quad a \equiv 1(\bmod 3), \\
p+2 a+x+3 y, & \text { if } \quad a \equiv-1(\bmod 3),
\end{array}\right.
\end{aligned}
$$




$$
12 \sum_{3}= \begin{cases}p+2 b-x-3 y, & \text { if } \quad a \equiv 1(\bmod 3) \\ p-2 b-x-3 y, & \text { if } \quad a \equiv-1(\bmod 3) .\end{cases}
$$

From (5.3) and (5.4) we obtain

(5.5) ind (3)

$$
\equiv\left\{\begin{array}{l}
1-\frac{1}{3}(2 a-2 b+x)-y+\frac{1}{6}(p-1)(\bmod 8), \text { if } a \equiv 1(\bmod 3), \\
1+\frac{1}{3}(2 a-2 b-x)-y+\frac{1}{6}(p-1)(\bmod 8), \text { if } \quad a \equiv-1(\bmod 3) .
\end{array}\right.
$$

Also, from Whiteman's tables, we have in every case,

$$
p+1-8 a+6 x \equiv 0(\bmod 16),
$$

so

ind (3)

$$
\begin{aligned}
& \equiv \begin{cases}1+2 a-2 b+\frac{p+1}{2}-4 a-y+\frac{1}{6}(p-1)(\bmod 8), & \text { if } a \equiv 1(\bmod 3), \\
1-2 a+2 b+\frac{p+1}{2}-4 a-y+\frac{1}{6}(p-1)(\bmod 8), & \text { if } a \equiv-1(\bmod 3),\end{cases} \\
& \equiv \begin{cases}-y(\bmod 8), & \text { if } a \equiv 1(\bmod 3), \\
4-y(\bmod 8), & \text { if } a \equiv-1(\bmod 3),\end{cases}
\end{aligned}
$$

which completes the proof of Theorem 4.

\section{REFERENCES}

1. Emma Lehmer, The quintic character of 2 and 3, Duke Math. J., 18 (1951), 11-18.

2. Criteria for cubic and quartic residuacity, Mathematika, 5 (1958), 20-29.

3. H. von Lienen, Primzahlen als achte Potenzreste, J. Reine Angew. Math., 266 (1974), 107-117.

4 J. B. Muskat, On the solvability of $x^{e} \equiv e(\bmod p)$, Pacific J. Math., $14(1964), 257-260$.

5. A. L. Whiteman, The cyclotomic number of order ten, Proceedings of the Symposia in Applied Mathematics 10, pp. 95-111, Amer. Math. Soc., Providence, Rhode Island, 1960 .

6. The cyclotomic numbers of order twelve, Acta Arith., 6 (1960), 53-76.

Received October 26, 1979. Research supported by the Natural Sciences and Engineering Research Council Canada Grant No. A-7233.

University of South Carolina

Columbia, SC 29208

AND

Carleton University

Ottawa, Ontario, Canada K1S 5B6 



\section{PACIFIC JOURNAL OF MATHEMATICS}

\section{EDITORS}

DoNALD BABBITT (Managing Editor)

University of Galifornia

Los Angeles, California 90024

HUgo RossI

University of Utah

Salt Lake City, UT 84112

C. C. MOORE AND ANDREW OGG

University of California

Berkeley, CA 94720
J. DugunduI

Department of Mathematics

University of Southern California

Los Angeles, California 90007

R. Finn and J. Milgram

Stanford University

Stanford, California 94305

\section{ASSOCIATE EDITORS}
E. F. BeCKenbaCh
B. H. NeumanN
F. WOLF
K. YoSHIDA

\section{SUPPORTING INSTITUTIONS}

UNIVERSITY OF BRITISH COLUMBIA

CALIFORNIA INSTITUTE OF TECHNOLOGY

UNIVERSITY OF CALIFORNIA

MONTANA STATE UNIVERSITY

UNIVERSITY OF NEVADA, RENO

NEW MEXICO STATE UNIVERSITY

OREGON STATE UNIVERSITY

UNIVERSITY OF OREGON
UNIVERSITY OF SOUTHERN CALIFONIA STANFORD UNIVERSITY UNIVERSITY OF HAWAII UNIVERSITY OF TOKYO UNIVERSITY OF UTAH WASHINGTON STATE UNIVERSITY UNIVERSITY OF WASHINGTON 


\section{Pacific Journal of Mathematics}

\section{Vol. 91, No. $1 \quad$ November, 1980}

Harvey Leslie Abbott, Extremal problems on nonaveraging and nondividing

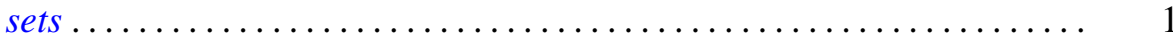

Marine Bruce Abrahamse and Stephen D. Fisher, Mapping intervals to

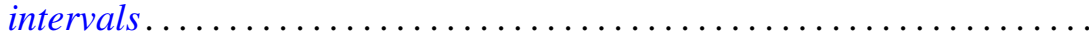

William Wells Adams, The best two-dimensional Diophantine

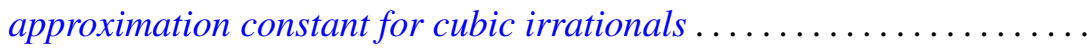

Marilyn Breen, A quantitative version of Krasnosel'skiu 's theorem in

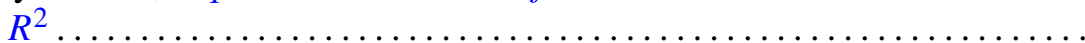

Stephen LaVern Campbell, Linear operators for which $T^{*} T$ and $T T^{*}$

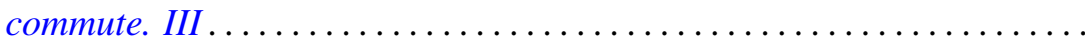

Zvonko Cerin, On cellular decompositions of Hilbert cube manifolds ......

J. R. Choike, Ignacy I. Kotlarski and V. M. Smith, On a characterization

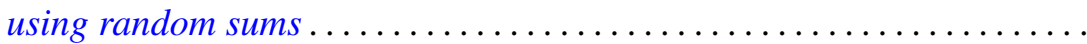

Karl-Theodor Eisele, Direct factorizations of measures .............. 79

Douglas Harris, Every space is a path component space ............. 95

John P. Holmes and Arthur Argyle Sagle, Analytic H-spaces, Campbell-Hausdorff formula, and alternative algebras.............

Richard Howard Hudson and Kenneth S. Williams, Some new residuacity criteria ..........................................

V. Karunakaran and Michael Robert Ziegler, The radius of starlikeness for a class of regular functions defined by an integral ....

Ka-Sing Lau, On the Banach spaces of functions with bounded upper

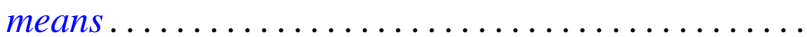

Daniel Paul Maki, On determining regular behavior from the recurrence formula for orthogonal polynomials................

Stephen Joseph McAdam, Asymptotic prime divisors and going down...

Douglas Edward Miller, Borel selectors for separated quotients ..

Kent Morrison, The scheme of finite-dimensional representations of an algebra

Donald P. Story, A characterization of the local Radon-Nikodým property by tensor products

Arne Stray, Two applications of the Schur-Nevanlinna algorithm ...

N. B. Tinberg, The Levi decomposition of a split $(B, N)$-pair ...

Charles Irvin Vinsonhaler and William Jennings Wickless, A theorem on quasi-pure-projective torsion free abelian groups of finite rank... 